\title{
Sagittal balance is more than just alignment: why PJK remains an unresolved problem
}

\author{
Steven D. Glassman, Mark P. Coseo and Leah Y. Carreon ${ }^{*}$
}

\begin{abstract}
Background: The durability of adult spinal deformity surgery remains problematic. Revision rates above $20 \%$ have been reported, with a range of causes including wound infection, nonunion and adjacent level pathology. While some of these complications have been amenable to changes in patient selection or surgical technique, Proximal Junctional Kyphosis (PJK) remains an unresolved challenge. This study examines the contributions of non-mechanical factors to the incidence of postoperative sagittal imbalance and PJK after adult deformity surgery.

Methods: We reviewed a consecutive series of adult spinal deformity patients who required revision for PJK from 2013 to 2015 and examined in their medical records in detail.

Results: Neurologic disorders were identified in 22 (76 \%) of the 29 PJK cases reviewed in this series. Neurologic disorders included Parkinson's disease (1), prior stroke (5), metabolic encephalopathy (2), seizure disorder (1), cervical myelopathy (7), thoracic myelopathy (1), diabetic neuropathy (5) and other neuropathy (4). Other potential comorbidities affecting standing balance included untreated cataracts (9), glaucoma (1) and polymyositis (1). Eight patients were documented to have frequent falls, with twelve cases having a fall right before symptoms related to the PJK were noted.

Conclusion: PJK is an important contributing factor to the substantial and unsustainable rate of revision surgery following adult deformity correction. Multiple efforts to avoid PJK via alterations in surgical technique have been largely unsuccessful. This study suggests that non-mechanical neuromuscular co-morbidities play an important role in post-operative sagittal imbalance and PJK. Recognizing the multi-factorial etiology of PJK may lead to more successful strategies to avoid PJK and improve surgical outcomes.
\end{abstract}

Keywords: Adult spinal deformity, Adult scoliosis, Proximal junctional kyphosis, PJK

\section{Background}

Surgical treatment of adult spinal deformity has progressed substantially over the past ten years. There have been significant advances in decision-making, medical management and surgical technique $[1,2]$. These improvements in evaluation and treatment have broadened the applicability of adult deformity surgery and lead to more reproducible clinical benefit based upon health related quality of life (HRQOL) scores [3, 4].

Despite these positive developments, the durability of adult spinal deformity surgery remains problematic.

\footnotetext{
* Correspondence: leah.carreon@nortonhealthcare.org Norton Leatherman Spine Center, 210 East Gray Street, Suite 900, Louisville, Kentucky 40202, USA
}

Revision rates above $20 \%$ have been reported, with a range of causes including wound infection, nonunion and adjacent level pathology [5-7]. While some of these complications have been amenable to changes in patient selection or surgical technique, Proximal Junctional yyphosis (PJK) remains an unresolved challenge.

The initial description of PJK in the pediatric literature was an increased sagittal angulation, without structural failure, at the upper aspect of a fusion construct [8]. At resent, the term is applied much more widely to delevel compression fracture, spondylolisthesis or fixation failure [6-10]. In general, this has been viewed as a 
consequence of poor bone quality, over-aggressive deformity correction or inadequate fixation.

PJK has been the focus of intense scrutiny, with multiple studies proposing mechanical solutions including adaptations for osteoporotic bone and in particular specific sagittal alignment targets [11, 12]. Unfortunately, none of these mechanical solutions have effectively decreased the rate of PJK. The role of this study is to examine the contributions of non-mechanical factors to the incidence of postoperative sagittal imbalance and PJK after adult deformity surgery.

\section{Methods}

After receiving Institutional Review Board Approval, we reviewed a consecutive series of adult spinal deformity patients who required revision for PJK from 2013 to 2015 and examined in their medical records in detail. Standard demographic data including age, gender, smoking status, height and weight were collected. Indications for the index surgery, specifics of the index surgery including upper instrumented vertebra fixation, time to PJK diagnosis, time to PJK surgery, mode of failure. Medical records were extensively evaluated for preoperative comorbidities; specifically for preoperative neurologic disorders and other pathologies that may affect standing balance.

\section{Results}

From 2012 to 2014, 245 patients underwent surgical correction of their adult spinal deformity at our institution. A true incidence of PJK will be difficult to determine as (1) some patients presenting at our institution with PJK had their index surgery performed elsewhere and (2) some of the patients who had their index surgery at our institution could have developed PJK and had surgery elsewhere.

Twenty-nine cases of $\mathrm{PJK}$ requiring revision were identified (Table 1). Of these 9 (31\%) were males and 10 (34 \%) were smokers. Mean age was 64.4 years. Mean BMI was $29 . \mathrm{kg} / \mathrm{m}^{2}$. Neurologic disorders were identified in $22(76 \%)$ of the PJK cases reviewed in this series. Neurologic disorders included Parkinson's disease (1), prior stroke (5), metabolic encephalopathy (2), seizure disorder (1), cervical myelopathy (7), thoracic myelopathy (1), diabetic neuropathy (5) and other neuropathy (4). Other potential comorbidities affecting standing balance included untreated cataracts (9), glaucoma (1) and polymyositis (1) (Table 2). Eight patients were documented to have frequent falls, with twelve cases having a fall right before symptoms related to the PJK were noted. Seventeen cases used an assistive device such as a cane, crutches or a walker and required a wheelchair. One patient had 5 co-morbid conditions affecting standing balance, two had 4 co-morbid conditions, four had 3 co- morbid conditions, nine had 2 co-morbid conditions and ten had only one co-morbid condition (Table 3).

\section{Discussion}

Proximal Junctional Kyphosis was first identified in 1999 [8], and was initially described as a radiographic finding with limited clinical relevance $[13,14]$. This sanguine assessment was short lived, as subsequent reports have documented the frequent need for revision surgery $[5,6]$ as well as the occurrence of catastrophic failures, termed Proximal Junctional Failure (PJF) $[9,10,15,16]$. The reported increase in PJK was coincident with several major changes in treatment paradigm. Adult deformity surgery became more common in older patients, and more aggressive correction was undertaken using osteotomies and rigid instrumentation. Studies have highlighted these factors and examined their etiologic role in PJK and PJF $[10,17,18]$.

Deformity surgeons clearly recognize PJK and PJF as important challenges, but often regard these complications as mechanical problems for which there should be a straight forward mechanical solutions. As osteoporosis is commonly identified as an etiology of PJK, surgeons have pursued options to offset poor bone quality. Strategies have included prophylactic medical treatment of low bone density, strengthening proximal instrumented and adjacent vertebral levels with cement injection. Other strategies have included decreasing rod rigidity, and softening the transition to unfused levels using hooks rather than screws [11, 19, 20]. Another major focus has been on selection of fusion levels and restoration of sagittal alignment [12, 18, 21, 22]. Studies have advocated both more aggressive and less aggressive deformity correction. Maruo et al. report that restoration of normal sagittal alignment protected against PJK, and that greater than 30-degree increase in lumbar lordosis was a significant risk factor for PJK. [18] As increase in lumbar lordosis is generally the mechanism by which normal sagittal alignment is restored, these observations appear contradictory.

The findings of the present study suggest that our failure to control the rate of PJK may be related in part to the narrow focus on mechanical factors. This study demonstrates that $76 \%$ of patients with PJK after spinal deformity correction have co-morbidities that adversely affect standing balance, regardless of alignment. These include neuromuscular disease, history of cerebral vascular accident, cervical myelopathy and neuropathy. All of these conditions may contribute to an inability to rebalance through unfused segments after deformity correction. This phenomenon is clearly recognized with substantial neurologic impairment such as patients with Parkinson's disease [23], but has not been clearly defined in those patients with less severe neurologic impairment. 
Table 1 Summary of cases

\begin{tabular}{|c|c|c|c|c|c|c|c|c|c|c|c|c|c|}
\hline $\begin{array}{l}\text { Case. } \\
\text { No. }\end{array}$ & $\begin{array}{l}\text { Age/ } \\
\text { Sex }\end{array}$ & Smoker & $\mathrm{BMI}$ & $\begin{array}{l}\text { Indication for } \\
\text { Index Surgery }\end{array}$ & Index Surgery & $\begin{array}{l}\text { UIV } \\
\text { Fixation }\end{array}$ & $\begin{array}{l}\text { Time to PJK } \\
\text { diagnosis }\end{array}$ & Mode of Failure & PJK surgery & $\begin{array}{l}\text { Fall } \\
\text { prior }\end{array}$ & $\begin{array}{l}\text { Assistive } \\
\text { devise }\end{array}$ & CCMI & Other co-morbidities \\
\hline 1 & $68 / F$ & Yes & 40.9 & Kyphoscoliosis & $\begin{array}{l}\text { PSF T10 to } \\
\text { Pelvis, TLIF L3-L4 }\end{array}$ & $\begin{array}{l}\text { bilateral } \\
\text { pedicle } \\
\text { screws }\end{array}$ & 8 months & $\begin{array}{l}\text { Fracture of T9-T10 } \\
\text { with cord } \\
\text { compression }\end{array}$ & $\begin{array}{l}\text { T9-T10 } \\
\text { laminectomy, } \\
\text { extension of fusion } \\
\text { T4-T11 }\end{array}$ & No & No & 11 & None \\
\hline 2 & $64 / M$ & Yes & 19.7 & Stenosis & PSF L3 to L5 & $\begin{array}{l}\text { bilateral } \\
\text { pedicle } \\
\text { screws }\end{array}$ & 18 months & Fracture of $\mathrm{L} 3$ & $\begin{array}{l}\text { PSO L3, PSF T11 to } \\
\text { pelvis }\end{array}$ & Yes & Cane & 11 & $\begin{array}{l}\text { CVA, Loss of reflexes below } \\
\text { knee }\end{array}$ \\
\hline 3 & $58 / \mathrm{M}$ & No & 33.9 & Multilevel stenosis & PSF L3 to Pelvis & $\begin{array}{l}\text { bilateral } \\
\text { pedicle } \\
\text { screws }\end{array}$ & 17 months & Fracture of $\mathrm{L} 3$ & $\begin{array}{l}\text { AIF L5-S1, Ponte } \\
\text { osteotomies, PSF } \\
\text { T10 to pelvis }\end{array}$ & Yes & No & 4 & CSM post ACDF \\
\hline 4 & $63 / F$ & No & 25.9 & Multilevel stenosis & PSF L2 to L5 & $\begin{array}{l}\text { bilateral } \\
\text { pedicle } \\
\text { screws }\end{array}$ & 21 months & $\begin{array}{l}\text { Compression of } L 2 \\
\text { with complete loss of } \\
\text { L1-L2 interspace }\end{array}$ & Extension to T10 & No & Wheelchair & 10 & $\begin{array}{l}\text { CVA, Cauda equina requiring } \\
\text { emergent decompression, } \\
\text { Diabetic neuropathy }\end{array}$ \\
\hline 5 & $65 / F$ & Yes & 34.9 & & $\begin{array}{l}\text { ASF L4-S1, PSF } \\
\text { T10 to Pelvis }\end{array}$ & $\begin{array}{l}\text { bilateral } \\
\text { pedicle } \\
\text { screws }\end{array}$ & 11 months & $\begin{array}{l}\text { Compression Fracture } \\
\text { T11 }\end{array}$ & $\begin{array}{l}\text { Extension of fusion } \\
\text { to } T 3\end{array}$ & Yes & Walker & 9 & $\begin{array}{l}\text { Diabetic neuropathy, } \\
\text { Frequent falls, post bilateral } \\
\text { TKA, ORIF L ankle }\end{array}$ \\
\hline \multirow[t]{2}{*}{6} & $70 / F$ & No & 25.7 & Kyphoscoliosis & $\begin{array}{l}\text { ASF, PSF T10 to } \\
\text { Pelvis }\end{array}$ & $\begin{array}{l}\text { bilateral } \\
\text { pedicle } \\
\text { screws }\end{array}$ & 12 months & $\begin{array}{l}\text { Compression Fracture } \\
\text { T9 }\end{array}$ & $\begin{array}{l}\text { Extension of fusion } \\
\text { to } T 3\end{array}$ & No & Cane & 7 & Cataracts \\
\hline & & & & & & & $\begin{array}{l}1 \text { month } \\
\text { after } 1 \text { st } \\
\text { PJK }\end{array}$ & $\begin{array}{l}\text { Pull out of claw } \\
\text { construct fracturing } \\
\text { T4 to T8 laminae }\end{array}$ & $\begin{array}{l}\text { Extension of Fusion } \\
\text { T2 to } T 12\end{array}$ & & & & \\
\hline 7 & $52 / F$ & Yes & 25.6 & $\begin{array}{l}\text { Degenerative } \\
\text { scoliosis, stenosis }\end{array}$ & $\begin{array}{l}\text { PSF, L2 to } \\
\text { sacrum }\end{array}$ & $\begin{array}{l}\text { bilateral } \\
\text { pedicle } \\
\text { screws }\end{array}$ & 64 months & $\begin{array}{l}\text { Kyphosis at L1-L2 im } \\
\text { pingement of screws } \\
\text { into disc space }\end{array}$ & $\begin{array}{l}\text { TLIF L1-L2, PSF L1- } \\
\text { L2 }\end{array}$ & Yes & Crutches & 8 & Diabetic neuropathy \\
\hline 8 & $64 / M$ & No & 31.0 & $\begin{array}{l}\text { Flatback S/P L3-L5 } \\
\text { PSF }\end{array}$ & $\begin{array}{l}\text { ASF L5-S1, PSF } \\
\text { T9 to Sacrum }\end{array}$ & $\begin{array}{l}\text { bilateral } \\
\text { pedicle } \\
\text { screws }\end{array}$ & 18 months & T8-T9 Listhesis & $\begin{array}{l}\text { Extension of fusion } \\
\text { to } T 2\end{array}$ & Yes & Walker & 8 & $\begin{array}{l}\text { CVA, Neuropathy, Cataracts } \\
\text { (removed), CSM post } \\
\text { laminectomy, Frequent falls, } \\
\text { post THA dislocation }\end{array}$ \\
\hline 9 & $57 / F$ & No & 30.5 & $\begin{array}{l}\text { Flatback S/P L3-L5 } \\
\text { laminectomies }\end{array}$ & PSF T11 to Pelvis & $\begin{array}{l}\text { bilateral } \\
\text { pedicle } \\
\text { screws }\end{array}$ & 25 months & $\begin{array}{l}\text { Compression Fracture } \\
\text { T9- T10 }\end{array}$ & $\begin{array}{l}\text { Extension of fusion } \\
\text { to } T 3\end{array}$ & No & No & 9 & $\begin{array}{l}\text { TIAs, Diabetic neuropathy, } \\
\text { Cataracts, Frequent falls, } \\
\text { post bilateral TKA, } \\
\text { multiple foot surgeries }\end{array}$ \\
\hline \multirow[t]{2}{*}{10} & $60 / M$ & Yes & 19.3 & Kyphoscoliosis & $\begin{array}{l}\text { ASF L4-S1, PSF } \\
\text { T10 to Pelvis }\end{array}$ & $\begin{array}{l}\text { bilateral } \\
\text { pedicle } \\
\text { screws }\end{array}$ & 82 months & $\begin{array}{l}\text { Fracture of T9, T8-T9 } \\
\text { spondylolisthesis }\end{array}$ & PSF T4 to T12 & Yes & Walker & 9 & $\begin{array}{l}\text { CVA, Sensory neuropathy, } \\
\text { Glaucoma, Frequent falls, } \\
\text { post multiple revisions } \\
\text { of bilateral TKA }\end{array}$ \\
\hline & & & & & & & $\begin{array}{l}14 \text { months } \\
\text { after } 1 \text { st } \\
\text { PJK }\end{array}$ & $\begin{array}{l}\text { Pull out of claw } \\
\text { construct fracturing } \\
\text { T3 lamina }\end{array}$ & $\begin{array}{l}\text { Extension of Fusion } \\
\mathrm{T} 1 \text { to } \mathrm{T} 10\end{array}$ & & & & \\
\hline 11 & $58 / \mathrm{M}$ & No & 34.7 & $\begin{array}{l}\text { Degenerative } \\
\text { scoliosis, stenosis }\end{array}$ & $\begin{array}{l}\text { PSF T10 to } \\
\text { Pelvis, TLIF L5-S1 }\end{array}$ & $\begin{array}{l}\text { bilateral } \\
\text { pedicle } \\
\text { screws }\end{array}$ & 1 month & T9-T10 Listhesis & PSF T4 to T10 & Yes & No & 6 & $\begin{array}{l}\text { DTs, Neuropathy, Frequent } \\
\text { falls, alcoholic, had DTs after } \\
\text { index surgery }\end{array}$ \\
\hline
\end{tabular}


Table 1 Summary of cases (Continued)

\begin{tabular}{|c|c|c|c|c|c|c|c|c|c|c|c|c|c|}
\hline$\overline{12}$ & $75 / F$ & No & 29.0 & $\begin{array}{l}\text { Degenerative } \\
\text { scoliosis S/P L2-L3 } \\
\text { PDSF }\end{array}$ & $\begin{array}{l}\text { PSF T10 to } \\
\text { Pelvis, TLIF L5-S1 }\end{array}$ & $\begin{array}{l}\text { bilateral } \\
\text { pedicle } \\
\text { screws }\end{array}$ & 27 months & Screw pull out & Extension to T4 & Yes & $\mathrm{No}$ & 5 & None \\
\hline \multirow[t]{2}{*}{13} & $62 / F$ & Yes & 30.0 & $\begin{array}{l}\text { Flatback deformity } \\
\text { S/P L2-LS1 PDSF }\end{array}$ & PSF L2 to S1 & $\begin{array}{l}\text { bilateral } \\
\text { pedicle } \\
\text { screws }\end{array}$ & 12 months & Fracture L1 & Extension to $\mathrm{T} 10$ & No & Cane & 6 & $\begin{array}{l}\text { Tremors, Multiple foot } \\
\text { surgeries }\end{array}$ \\
\hline & & & & & & & $\begin{array}{l}38 \text { months } \\
\text { after } 1 \mathrm{st} \\
\text { PJK }\end{array}$ & $\begin{array}{l}\text { T9-T10 fracture with } \\
\text { erosion of screws } \\
\text { into disc }\end{array}$ & $\begin{array}{l}\text { Removal of } \\
\text { instrumentation, } \\
\text { PSF T4 to L2 }\end{array}$ & & & & \\
\hline 14 & $69 / F$ & Yes & 26.5 & $\begin{array}{l}\text { Adjacent segment } \\
\text { degeneration S/P L3 } \\
\text { to L5 PSF }\end{array}$ & $\begin{array}{l}\text { Extension of } \\
\text { fusion L1 to S1 }\end{array}$ & $\begin{array}{l}\text { bilateral } \\
\text { pedicle } \\
\text { screws }\end{array}$ & 1 month & L1-L2 listhesis & $\begin{array}{l}\text { Extension from } \mathrm{T} 10 \\
\text { to S1 }\end{array}$ & No & No & 7 & None \\
\hline 15 & $57 / F$ & No & 40.8 & $\begin{array}{l}\text { Degenerative } \\
\text { scoliosis, stenosis }\end{array}$ & PSF L2 to S1 & $\begin{array}{l}\text { bilateral } \\
\text { pedicle } \\
\text { screws }\end{array}$ & 92 months & L1-L2 listhesis & $\begin{array}{l}\text { Extension from } \mathrm{T} 10 \\
\text { to S1 }\end{array}$ & No & Cane & 5 & Parkinson's disease \\
\hline 16 & $63 / F$ & No & 23.2 & $\begin{array}{l}\text { Adjacent segment } \\
\text { stenosis S/P L1 to S1 } \\
\text { PSDF }\end{array}$ & PSF T9 to L3 & $\begin{array}{l}\text { bilateral } \\
\text { pedicle } \\
\text { screws }\end{array}$ & 7 months & $\begin{array}{l}\text { Posterior lysis of T9 } \\
\text { and } 110\end{array}$ & $\begin{array}{l}\text { Removal of } \\
\text { instrumentation, } \\
\text { PSF T4 to L3 }\end{array}$ & Yes & Cane & 8 & $\begin{array}{l}\text { Cataract, CSM post C } 3 \text { to } \mathrm{T} 1 \\
\text { ACDF }\end{array}$ \\
\hline 17 & $73 / F$ & No & 36.4 & Scoliosis & $\begin{array}{l}\text { PSF T6 to } \\
\text { Sacrum }\end{array}$ & $\begin{array}{l}\text { bilateral } \\
\text { hooks }\end{array}$ & 80 months & Fracture T6 & $\begin{array}{l}\text { Removal of } \\
\text { instrumentation, } \\
\text { PSF T3 to L1 }\end{array}$ & No & No & 9 & Cataract \\
\hline 18 & $61 / M$ & Yes & 32.5 & Scoliosis & $\begin{array}{l}\text { PSF T8 to } \\
\text { Sacrum }\end{array}$ & $\begin{array}{l}\text { bilateral } \\
\text { pedicle } \\
\text { screws }\end{array}$ & 11 months & Fracture 78 & $\begin{array}{l}\text { Removal of } \\
\text { instrumentation, } \\
\text { PSF T4 to Pelvis }\end{array}$ & No & Walker & 7 & Polymyositis \\
\hline \multirow[t]{2}{*}{19} & $72 / M$ & No & 36.8 & Scoliosis & PSF T11 to L3 & $\begin{array}{l}\text { bilateral } \\
\text { pedicle } \\
\text { screws }\end{array}$ & 23 months & T10-T11 listhesis & $\begin{array}{l}\text { Removal of } \\
\text { instrumentation, } \\
\text { PSF T8 to T11 }\end{array}$ & No & Walker & 6 & $\begin{array}{l}\text { CSM post laminoplasty, } \\
\text { Frequent falls }\end{array}$ \\
\hline & & & & & & & $\begin{array}{l}41 \text { months } \\
\text { after } 1 \mathrm{st} \\
\text { PJK }\end{array}$ & T7-T8 listhesis & $\begin{array}{l}\text { Removal of } \\
\text { instrumentation T9- } \\
\text { L1, PSF T2 to T9 }\end{array}$ & & & & \\
\hline 20 & $78 / F$ & No & 39.9 & Scoliosis & PSF L1 to S1 & $\begin{array}{l}\text { bilateral } \\
\text { pedicle } \\
\text { screws }\end{array}$ & 14 months & Fracture T12 & $\begin{array}{l}\text { Extension of Fusion } \\
\text { to T8 }\end{array}$ & No & No & 7 & $\begin{array}{l}\text { Metabolic encephalopathy, } \\
\text { Cataract }\end{array}$ \\
\hline 21 & $71 / F$ & Yes & 26.5 & $\begin{array}{l}\text { Degenerative } \\
\text { Scoliosis }\end{array}$ & PSF L2-L3 & $\begin{array}{l}\text { bilateral } \\
\text { pedicle } \\
\text { screws }\end{array}$ & 45 months & $\begin{array}{l}\text { L1-L2 collapse and } \\
\text { localized scoliosis }\end{array}$ & Extension to $\mathrm{T} 10$ & No & Cane & 10 & $\begin{array}{l}\text { Cataract (removed), Cervical } \\
\text { osteomyelitis with cord } \\
\text { compromise }\end{array}$ \\
\hline 22 & $75 / F$ & No & 30.4 & Scoliosis & PSF T4 to Pelvis & $\begin{array}{l}\text { bilateral } \\
\text { hooks }\end{array}$ & 2 months & $\begin{array}{l}\text { Hook pull-out with } \\
\text { T4-T6 laminar } \\
\text { fractures }\end{array}$ & Extension to T2 & No & No & 10 & Cataract \\
\hline 23 & $69 / M$ & No & 27.4 & $\begin{array}{l}\text { Post-laminectomy } \\
\text { instability }\end{array}$ & $\begin{array}{l}\text { ASF } L 3 \text { to } S 1 \\
\text { PSF } L 2 \text { to } S 1\end{array}$ & $\begin{array}{l}\text { bilateral } \\
\text { pedicle } \\
\text { screws }\end{array}$ & 1 month & $\begin{array}{l}\text { Compression Fracture } \\
\text { of } L 2 \text { with screw } \\
\text { pullout }\end{array}$ & $\begin{array}{l}\text { Removal of } \\
\text { instrumentation, } \\
\text { PSF T10 to L1 }\end{array}$ & No & No & 6 & Diabetic neuropathy \\
\hline 24 & $55 / F$ & No & 38.0 & & & & 9 months & $\begin{array}{l}\text { Compression Fracture } \\
\mathrm{T} 10\end{array}$ & Extension to T3 & Yes & No & 8 & CSM post ACDF \\
\hline
\end{tabular}


Table 1 Summary of cases (Continued)

\begin{tabular}{|c|c|c|c|c|c|c|c|c|c|c|c|c|c|}
\hline & & & & $\begin{array}{l}\text { Adjacent segment } \\
\text { stenosis S/P L2 to } \\
\text { S1 PSDF }\end{array}$ & $\begin{array}{l}\text { PSF T10 to } \\
\text { Pelvis, TLIF L2- } \\
\text { L3, L5-S1 }\end{array}$ & $\begin{array}{l}\text { bilateral } \\
\text { pedicle } \\
\text { screws }\end{array}$ & & & & & & & \\
\hline \multirow[t]{2}{*}{25} & $70 / F$ & No & 35.5 & Stenosis & PDSF L2-L5 & $\begin{array}{l}\text { bilateral } \\
\text { pedicle } \\
\text { screws }\end{array}$ & 10 months & Compression of $L 2$ & PSF T10 to Pelvis & Yes & Cane & 7 & $\begin{array}{l}\text { Metabolic encephalopathy, } \\
\text { Cataract, Frequent falls }\end{array}$ \\
\hline & & & & & & & $\begin{array}{l}5 \text { months } \\
\text { after 1st } \\
\text { PJK }\end{array}$ & $\begin{array}{l}\text { Compression Fracture } \\
\text { T9 }\end{array}$ & Extension to $\mathrm{T} 2$ & & & & \\
\hline 26 & $62 / F$ & No & 21.4 & $\begin{array}{l}\text { Adjacent segment } \\
\text { stenosis S/P L3 to } \\
\text { L5 PSDF }\end{array}$ & $\begin{array}{l}\text { ASF } L 2 \text { to } L 5, \\
\text { Extension of } \\
\text { fusion to } T 10\end{array}$ & $\begin{array}{l}\text { bilateral } \\
\text { hooks }\end{array}$ & 3 months & Fracture T10 & Extension to $\mathrm{T} 2$ & No & Cane & No & $\begin{array}{l}\text { CSM post ACDF, Neuropathy, } \\
\text { Frequent falls }\end{array}$ \\
\hline 27 & $73 / F$ & No & 20.6 & Scoliosis & PSF T10 to Pelvis & $\begin{array}{l}\text { bilateral } \\
\text { pedicle } \\
\text { screws }\end{array}$ & 4 months & $\begin{array}{l}\text { T10 compression } \\
\text { fracture }\end{array}$ & PSF T7 to T12 & Yes & Walker & Yes & $\begin{array}{l}\text { Mild cognitive impairment, } \\
\text { Benign thoracic tumor S/P } \\
\text { excision }\end{array}$ \\
\hline 28 & $65 / F$ & No & 21.1 & Scoliosis & PSF T11 to S1 & $\begin{array}{l}\text { bilateral } \\
\text { pedicle } \\
\text { screws }\end{array}$ & 22 months & $\begin{array}{l}\text { T10-T11 listhesis, } \\
\text { nonunion L5-S1 }\end{array}$ & $\begin{array}{l}\text { AIF L3 to S1, PSF } \\
\text { T10 to Pelvis }\end{array}$ & No & No & No & Seizures, Eye surgery \\
\hline 29 & $33 / M$ & Yes & 26.7 & Scoliosis & PSF L1 TO L4 & $\begin{array}{l}\text { bilateral } \\
\text { pedicle } \\
\text { screws }\end{array}$ & 22 months & Compression of $\mathrm{T} 12$ & $\begin{array}{l}\text { Removal of } \\
\text { instrumentation, } \\
\text { PSF T10 to Pelvis }\end{array}$ & No & Cane & No & Chronic dropfoot \\
\hline
\end{tabular}

PSDF posterior spinal decompression and fusion, PSF posterior spinal fusion, ASF anterior spinal fusion, TLIF transforaminal lumbar interbody fusion, CVA cerebrovascular accident, CSM cervical spondylotic myelopathy, $A C D F$ anterior cervical discectomy and fusion, $T K A$ total knee arthroplasty, ORIF open reduction internal fixation, $T H A$ total hip arthroplasty, $D T$ delirium tremens 
Table 2 Frequency of co-morbid conditions that can affect

\begin{tabular}{ll} 
balance & Frequency \\
\hline Co-morbid condition & 5 \\
\hline Prior stroke & 2 \\
Metabolic encephalopathy & 1 \\
Parkinson's disease & 1 \\
Seizures & 1 \\
Polymyositis & 5 \\
Diabetic Neuropathy & 4 \\
Neuropathy & 9 \\
Cataract & 1 \\
Glaucoma & 8 \\
Myelopathy & 8 \\
Frequent falls &
\end{tabular}

Beyond potential neurogenic causes of standing imbalance, other factors such as visual impairment, vestibular dysfunction and severe muscular deconditioning also impact balance and gait [24, 25]. Visual impairment was noted in $40 \%$ of PJK cases and more than a single potentially relevant co-morbidity was noted in more than $66 \%$ of cases. While these findings do not implicate neuromuscular disease as the direct cause of PJK, they certainly suggest a multi-factorial etiology.

The mechanisms by which these non-mechanical risk factors contribute to PJK are not well defined, and probably do not represent a unique common pathway. In some instances, such as patients with neuropathy or central neurologic deterioration, an impaired feedback loop may limit the ability to compensate appropriately after mechanical realignment. In essence, the patient's brain does not properly register the "improved alignment" as determined by radiographic assessment. In other cases, lack of appropriate sensory feedback may result in accelerated proximal segment degeneration, akin to the appearance of a Charcot joint. In patients with severe deconditioning, muscular support may be inadequate regardless of mechanical alignment.

It is not completely clear how best to apply these observations in clinical practice. Our case series methodology cannot provide a relative risk assessment for any

Table 3 Number of co-morbid conditions that can affect balance

\begin{tabular}{ll}
\hline & Frequency \\
\hline None & 3 \\
One & 10 \\
Two & 9 \\
Three & 4 \\
Four & 2 \\
Five & 1 \\
\hline
\end{tabular}

of the individual co-morbid conditions, and to-date no diagnostic test has been developed to quantify a global risk for post-operative standing imbalance or PJK. It is also unknown as to whether these risks can be modified by pre-operative interventions such as balance training, in the same way that treatment of osteoporosis is thought to reduce the risk of post-operative vertebral fracture or screw pull-out.

Weaknesses of this study include firstly the case series methodology. As some of the patients had their index procedure elsewhere, we do not have an accurate denominator to assess the incidence of PJK in the primary cohort. This series is also relatively small, so that the relative risk of the various co-morbidities cannot be effectively compared. Despite these weaknesses, this study clearly supports the role of concomitant neuromuscular disease in the development of post-op standing imbalance and PJK. The data does not provide a specific threshold at which surgery should be withheld, but certainly emphasizes the importance of including an assessment of associated neuromuscular disease in preoperative planning and shared decision-making.

Spine surgeons have devoted a great deal of time and effort to defining optimal sagittal alignment, but sagittal balance is more than just alignment. Dubousset outlined the many interactive systems that contribute to ambulation and stated, "good alignment is preferable in order to obtain a good balance, but it is not sufficient" [26]. Understanding and avoiding PJK requires that we move beyond the one-dimensional view that finding an ideal sagittal alignment, softening the transition at the proximal aspect of the instrumented segment, or improving the adjacent bone strength will solve the problem of PJK. Thinking about PJK more broadly is a step in the right direction.

\section{Conclusions}

PJK is an important contributing factor to the substantial and unsustainable rate of revision surgery following adult deformity correction. Multiple efforts to avoid PJK via alterations in surgical technique have been largely unsuccessful. This study suggests that non-mechanical neuromuscular co-morbidities play an important role in post-operative sagittal imbalance and PJK. Recognizing the multi-factorial etiology of PJK may lead to more successful strategies to avoid PJK and improve surgical outcomes.

\footnotetext{
Competing interests

SDG - is an employee of Norton Healthcare; received research grants from Norton Healthcare; holds patents from and receives royalties from Medtronic Medtronic provided funds directly to database company. No funds were paid directly to Individual or Individual's Institution 01/2002 to 09/2009. Nuvasive provided funds directly to database company. No funds are paid directly to Individual or Individual's Institution 06/2012-04/2015. MPK has nothing to disclose.
} 
LYC - is an employee of Norton Healthcare; Member, Editorial Advisory Board, Spine and Spine Journal; Institutional Review Board Member, University of Louisville Institutional Review Board; Global Evidence Advisory Board Member, Medtronic 2012, 2013; Research Committee Member, Scoliosis Research Society; received research grants from Norton Healthcare and AO Spine paid directly to Scoliosis Research Society 2010-2011; receives research funds from the Orthopedic Research and Educational Fund, 2013present; received research grant from Norton Healthcare, 2013; receives Scoliosis Research Society Research Funding, 2013-present; receives research funding from the Norton Healthcare James R. Petersdorf Fund 2015; received funds for travel for Study Planning Meetings from Orthopedic Educational Research Fund, Department of Defense, Association for Collaborative Spine Research and the Center for Spine Surgery and Research of the University of Southern Denmark; received funds for travel for Annual required Continuing Education for Institutional Review Board Members, University of Louisville Institutional Review Board; Honoraria for participation in Review Panels National Institutes of Health, Children's Tumor Foundation, and Global Evidence Advisory Board, Medtronic. Medtronic provided funds directly to database company. No funds were paid directly to Individual or Individual's Institution 01/2002 to 09/2009. Nuvasive provided funds directly to database company. No funds are paid directly to Individual or Individual's Institution 06/2012-04/2015.

\section{Authors' contributions}

SDG - conception and design, analysis and interpretation of data, drafting of the manuscript, acquisition of data, MPC - acquisition of data, analysis and interpretation of data, critical revision of the manuscript, LYC - acquisition of data, analysis and interpretation of data, critical revision of the manuscript. All authors read and approved the final manuscript.

\section{Acknowledgments}

No other person aside from the authors made substantial contributions to conception, design, acquisition of data, or analysis and interpretation of data, or was involved in drafting the manuscript or revising it critically for important intellectual content. No funding was received for the design, in the collection analysis, and interpretation of data; in the writing of the manuscript; and in the decision to submit the manuscript for publication. No language editor or scientific (medical) writer was involved in the preparation of the manuscript.

\section{Disclaimer}

The views expressed in this presentation are those of the author and do not necessarily reflect the official policy or position of the Department of the Navy, Department of Defense, or the United States government.

Received: 5 November 2015 Accepted: 4 January 2016

Published online: 22 January 2016

\section{References}

1. Schwab FJ, Lafage V, Farcy JP, Bridwell KH, Glassman S, Shainline M. Predicting outcome and complications in the surgical treatment of adult scoliosis. Spine. 2008:33:2243-7.

2. Smith JS, Shaffrey Cl, Berven S, Glassman S, Hamill C, Horton W, et al. Operative versus nonoperative treatment of leg pain in adults with scoliosis A retrospective review of a prospective multicenter database with two-year follow-up. Spine. 2009;34:1693-8.

3. Bridwell KH, Glassman S, Horton W, Shaffrey C, Schwab F, Zebala LP, et al. Does treatment (nonoperative and operative) improve the two-year quality of life in patients with adult symptomatic lumbar scoliosis? A prospective multicenter evidence-based medicine study. Spine. 2009;34:2171-8.

4. Ledonio CGT, Polly DW, Crawford CH, Duval S, Smith JS, Buchowski JM, et al. Adult degenerative scoliosis surgical outcomes: A systematic review and meta-analysis. Spine Deformity. 2013;1:248-58.

5. Glassman SD, Dimar JR, Carreon LY. Revision rate after adult deformity surgery. Spine Deformity. 2015;3:199-203.

6. Lau D, Clark AJ, Scheer JK, Daubs MD, Coe JD, Paonessa KJ, et al. Proximal junctional kyphosis and failure after spinal deformity surgery. Spine. 2014;30:2093-102.

7. Yagi $\mathrm{M}$, Akilah $\mathrm{KB}$, Boachie-Adjei $\mathrm{O}$. Incidence, risk factors and classification of proximal junctional kyphosis: Surgical outcomes review of adult idiopathic scoliosis. Spine. 2010;36:E60-8.
8. Lee GA, Betz RR, Clements 3rd DH, Huss GK. Proximal kyphosis after posterior spinal fusion in patients with idiopathic scoliosis. Spine. 1999;24:795-9.

9. Hart RA, McCarthy I, Ames CP, Shaffrey Cl, Hamilton DK, Hostin R. Proximal junctional kyphosis and proximal junctional failure. Neurosurg Clin N Am. 2013;24:213-8.

10. Hostin R, McCarthy I, O'Brien M, Bess S, Line B, Boachie-Adjei O, et al. Incidence, mode, and location of acute proximal junctional failures after surgical treatment of adult spinal deformity. Spine (Phila Pa 1976). 2013;38(12):1008-15

11. Martin CT, Skolasky RL, Mohamed AS, Kebaish KM. Preliminary results of the effect of prophylactic verbetroplasty on the incidence of proximal junctional complications after posterior spinal fusion to the low thoracic spine. Spine Deformity. 2013;1:132-8.

12. Schwab F, Patel A, Ungar B, Farcy JP, Lafage V. Adult spinal deformitypostoperative standing imbalance. Spine. 2010;35:2224-31.

13. Glattes RC, Bridwell KH, Lenke LG, Kim YJ, Rinella A, Edwards C. Proximal junctional kyphosis in adult spinal deformity following long instrumented posterior spinal fusion. Spine. 2005;30:1643-9.

14. Kim YJ, Lenke LG, Bridwell KH, Kim J, Cho SK, Cheh G, et al. Proximal junctional kyphosis in adolescent idiopathic scoliosis after 3 different types of posterior segmental spinal instrumentation and fusions. Incidence and risk factor analysis of 410 cases. Spine. 2007;32:2731-8.

15. O'Leary PT, Bridwell KH, Lenke LG, Good CR, Pichelmann MA, Buchowski JM, et al. Risk factors and outcomes for catastrophic failures at the top of long pedicle screw constructs. A matched cohort analysis performed at a single center. Spine. 2009;34:2134-9.

16. Watanabe K, Lenke LG, Bridwell KH, Kim YJ, Koester L, Hensley M. Proximal junctional vertebral fracture in adults after spinal deformity surgery using pedicle screw constructs. Spine. 2010;35:138-45.

17. Kim YJ, Bridwell KH, Lenke LG, Rhim S, Cheh G. Pseudoarthrosis in long adult spinal deformity instrumentation and fusion to the sacrum: Prevalence and risk factor analysis of 144 cases. Spine. 2006:31:2329-36.

18. Maruo K, Ha Y, Inoue S, Samuel S, Okada E, Hu SS, et al. Predictive factors for proximaljunctional kyphosis in long fusions to the sacrum in adult spinal deformity. Spine (Phila Pa 1976). 2013;38((23):E1469-76.

19. Kebaish KM, Martin CT, O'Brien JR, LaMotta IE, Voros GD, Belkoff SM. Use of vertebroplasty to prevent proximal junctional fractures in adult deformity surgery: a biomechanical cadaveric study. Spine J. 2013;13:1897-903.

20. Theologis AA, Burch S. Prevention of acute proximal junctional fractures after long thoracolumbar posterior fusions for adult spinal deformity using 2-level cement augmentation at the upper instrumented vertebra and the vertebral 1 level proximal to the upper instrumented vertebra. Spine. 2015:40:1516-26.

21. Kim HJ, Bridwell KH, Lenke LG, Park MS, Song KS, Piyaskulkaew C, et al. Patients with proximal junctional kyphosis requiring revision surgery have higher postoperative lumbar lordosis and larger sagittal balance corrections. Spine. 2014:39:E576-80.

22. Kim YJ, Bridwell KH, Lenke LG, Rhim S, Cheh G. Sagittal thoracic decompensation following long adult lumbar spinal instrumentation and fusion to L5 or S1: Causes, prevalence, and risk factor analysis. Spine. 2006 31:2359-66.

23. Upadhyay CD, Starr PA, Mummaneni PV. Spinal deformity and Parkinson disease: a treatment algorithm. Neurosurg Focus. 2010;28:E5.

24. Aartolahti E, Häkkinen A, Lönnroos E, Kautiainen H, Sulkava R, Hartikainen S. Relationship between functional vision and balance and mobility performance incommunity-dwelling older adults. Aging Clin Exp Res. 2013;25(5):545-52

25. Willis JR, Vitale SE, Agrawal Y, Ramulu PY. Visual impairment, uncorrected refractive error, and objectively measured balance in the United States. JAMA Ophthalmol. 2013:131(8):1049-56.

26. Dubousset J, Challier V, Farcy JP, Schwab FJ, Lafage V. Spinal alignment versus spinal balance. In: Haid RW, Schwab FJ, Shaffrey Cl, Youssef JA, editors. Global Spinal Alignment: Principles, Pathologies, and Procedures. St. Louis, MO: Quality Medical Publishing; 2015. p. 3-9. 